Acta vet. scand. 1997, 38, 201-205.

Brief Communication

\title{
Occurrence of Pneumocystis carinii in Canine Distemper
}

Pneumocystis carinii is a eukaryotic opportunistic pathogen causing pneumonia (PCP) in immunosuppressed patients. It is best known in human medicine as a pathogen of AIDS patients and in immunosuppressed transplant and cancer patients (Waltzer 1993).

The only known part of the life-cycle takes place in mammalian lung alveolus where $P$. $\mathrm{Ca}$ rinii can be found in two main forms - as trophozoites or as cysts. In histopathology of PCP, alveoli are filled with eosinophilic honeycomblike material containing tissue fluid, exudated serum and abundant number of trophozoites and cysts. As a nonspecific response to alveolar injury type II pneumocytes show hypertrophy and proliferation. Histological changes are revealed in severe hypoxemia and increased alveolar-arterial oxygen differences are seen in arterial blood gas analysis. In thoracic radiography, diffuse interstitial and peribronchial densities could be seen in a clinical canine case (Sukura et al. 1996). Specific PCP diagnosis in dogs can be made by demonstrating $P$. carinii organisms in lung-originated specimens; however, $P$ carinii organisms can be found also in the lungs of apparently healthy individuals (Bartlett \& Smith 1991). Therefore, it has been debated whether a clinical $P$. carinii infection is due to reactivation of latent infection or to infection de novo.

Canine distemper virus (CDV) infection results in viral strain dependent immunosuppression and lymphopenia of both B and T cells (Krakowa et al. 1980). Recently, it has been shown in natural infection, that CD4+ lymphocytes are major target cells for CDV (Iwatsuki et al. 1995). Respectively, in AIDS-patients, decreased circulating CD4+ T-cell count in peripheral blood is a well documentated risk factor for the development of PCP (Phair et al. 1990). Only few reports have been made of PCP in dogs, most commonly among young animals (Farrow et al. 1972, Furuta et al. 1994, Botha \& Van Rensburg 1979, Sukura et al. 1996). As a clinical disease, it often has a fatal outcome. Among domestic animals a clinical disease state has been described in horses, pigs and goats (McConnell et al. 1971, Yoshida \& Ikai 1979, Shimizu et al. 1985, Bille-Hansen et al. 1990, Ainsworth et al. 1993). PCP due to congenital immunodeficiency in domestic animals is best known in Arabian foals, which suffer from severe combined immunodeficiency (Perryman et al. 1978, Mayhew \& Greiner 1986). There is also some indirect evidence of an inheritable immunodeficiency in dogs which might predispose them to PCP (Farrow et al. 1972). However, a similar association to such viral infections as HIV and PCP has not been reported in domestic animals.

All histologically and/or virologically confirmed canine distemper (CD) cases from 1990 to 1992 were retrospectively collected from the database of the Pathology Unit, National Vete- 
rinary and Food Research Institute (NVFRI). If paraffin-embedded lung specimens were available, they were included in this study as CD cases. Non-CD cases were collected from the NVFRI and from the Department of Pathology, Faculty of Veterinary Medicine, University of Helsinki. Selection criteria of non-CD cases were: young dog without evident immunosuppression.

Lung specimens $(\mathrm{N}=85)$ were fixed in $10 \%$ neutral buffered formalin. Fixed tissues were dehydrated, embedded in paraffin and sectioned at $5 \mu \mathrm{m}$. From each animal several lung sections were stained with Grocott's modification of Gomori's Methenamine Silver stain (GMS) (Grocott 1955). To optimize incubation times positive control specimens of rat origin were included in all stainings. The slides were studied by experienced investigators at a magnification of $200 x$ or $400 x$. The animal was considered

$P$ carinii-positive if more than 5 cysts were found per slide. Comparisons of proportions were statistically analyzed with Fisher's exact test.

All the CD cases were examined at NVFRI. Paraffin blocks of lung specimens were available in 35 dogs with distemper, which had died or had been euthanized during the study period. In 3 samples information on sex was missing in the database, and the rest of the dogs comprised equal numbers of males $(\mathrm{N}=16)$ and females $(\mathrm{N}=16)$ (Table 1).

Non-CD dogs died due to trauma or dysontogenesis: 18 such cases were found in the NVFRI and 32 from the Faculty of Veterinary Medicine. Samples for canine distemper virus antigens were studied and found to be negative only occasionally in non-CD controls, but histologically they were not diagnosed to have $\mathrm{CD}$ infection.

Most of the dogs studied were purebred (76/85), all $P$. carinii-positive CD cases be-
Table 1. Results and anamnestic data of P. carinii investigation of canine distemper-infected and noninfected dogs.

\begin{tabular}{lcc}
\hline & CD-cases & Non CD-cases \\
\hline P.carinii-positive & 5 & 0 \\
Mean age (month) & 8.7 & 8.1 \\
Range & $0.5-72$ & $2-30$ \\
Female & 16 & 22 \\
Male & 16 & 27 \\
No information of sex & 3 & 1 \\
Total & 35 & 50 \\
\hline
\end{tabular}

longed to different breeds: Finnish Harrier, Hovawart, Toy Poodle, Bearded Collie and Great Dane.

Five of the $35 \mathrm{CD}$ cases were also $P$. cariniipositive (14\%), while none of the non CD cases turned out to harbour any $P$. carinii cysts $(5 / 35$ vs $0 / 50, \mathrm{p}<0.01)$. Two of the 5 P. carinii-positive animals (Fig. 1) also had histologically confirmed pneumonia. However, this frequency $(2 / 5)$ did not differ from the frequency of pneumonia in other $C D$ cases $(7 / 30 ; p=0.59)$.

In Finland, after a long period during which no cases of canine distemper had been reported, clinical cases began to occur again in the beginning of the 1990s and, thereafter, with an epidemic pattern divided into 2 phases, 1990-92 and 1994-95. An association of PCP with viral disease, similar to the association between HIV and PCP in man, has been reported in monkeys (Baskerville et al. 1991, Vogel et al. 1993), but not in domestic animals. Feline immunodeficiency virus has many similarities to HIV ( $\mathrm{Pe}$ dersen 1993) and has, therefore, been used as a model for HIV in order to study the pathogenesis of AIDS (Gardner \& Luciw 1990); but, as far we know, no one has reported any occurrence of PCP in FAIDS (feline acquired immunodeficiency syndrome) cats. The one survey seeking any eventual association between feline 


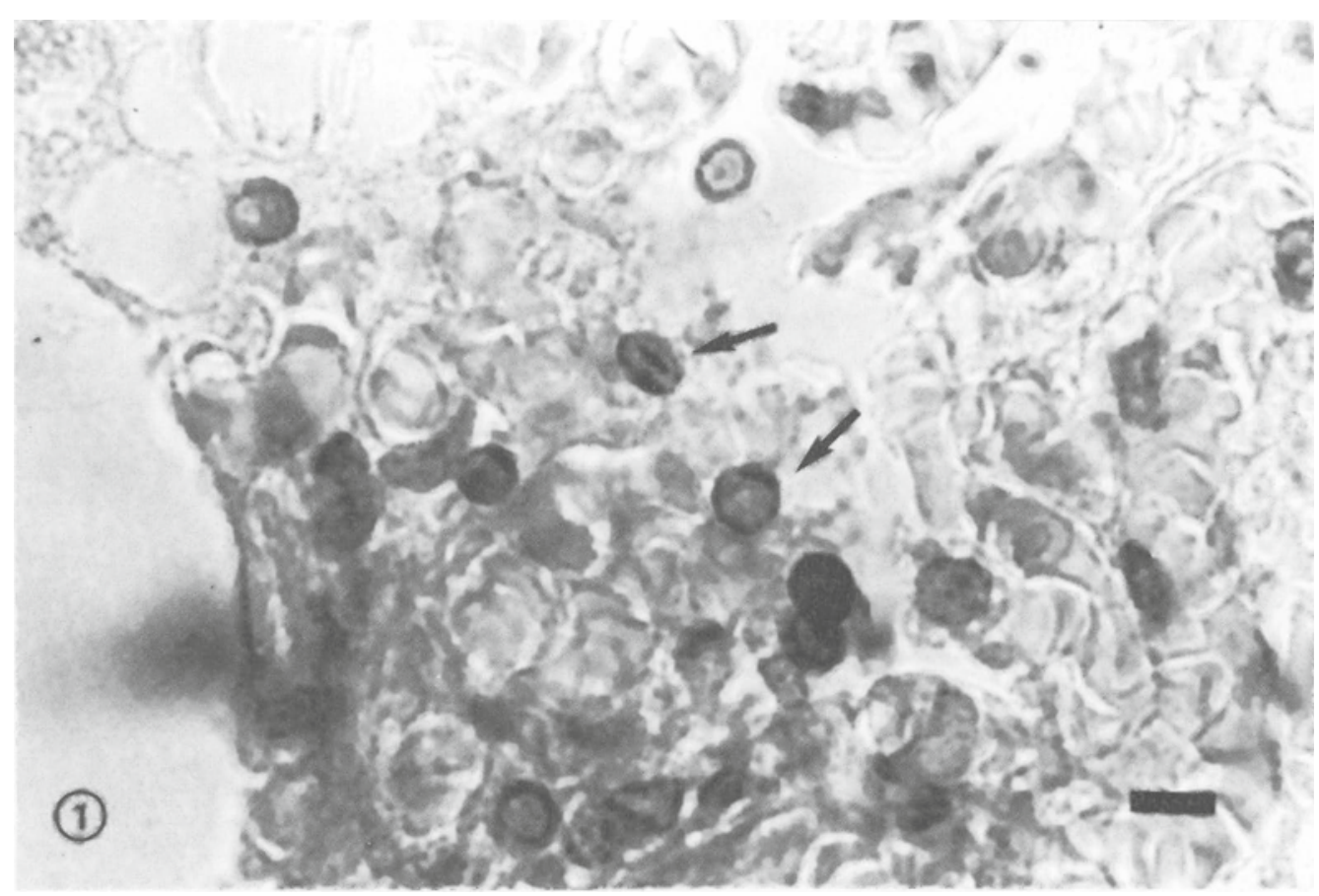

Figure 1. Lung section of canine distemper virus-positive dog showing cyst forms of Pneumocystis carinii (arrows). The animal had a histologically confirmed pneumonia but the histopathological changes were not typical for Pneumocystis carinii pneumonia (PCP). Hematoxylin-eosin and methenamine silver stains, bar $=5 \mu \mathrm{m}$.

pressive viral infection in the cat - and PCP could show no $P$. carinii in any of its FeLV-infected cats (Hagler et al. 1987).

$\mathrm{CD}$ virus is considered to be immunosuppressive (Krakowa et al. 1980), but with cross-sectional study design it is impossible to deduce whether the higher prevalence of $P$. carinii in $\mathrm{CD}$ cases was due to the immunosuppressive effect of CD infection, or whether the $C D$ was due to some underlying immunodeficiency which also allowed manifestation of $P$. carinii. In $\mathrm{CD}$ infection, secondary respiratory infections are very common. However, in our material the frequency of pneumonia in $\mathrm{CD}+/ \mathrm{PC}+$ dogs did not differ from that in other CD-infected dogs.

$P$ carinii organisms can exist in the lungs with- out causing a clinical disease, and latent or subclinical infection seems to be relatively common in some wild mammalian species (Laakkonen \& Soveri 1995). The prevalence study in Denmark found one of $106(0.9 \%)$ healthy dogs to be positive for $P$. carinii organisms (Settnes \& Hasselager 1984), and a Japanese study reported 2 of 13 studied dogs to be positive (Shimizu et al. 1985), but the health status of the dogs was not recorded. Most reported PCP cases in dogs have been in miniature Dachshunds (Botha \& Van Rensburg 1979). In our material, none of the P. carinii-positive animals were a Dachshund. There was only one Dachshund among the $\mathrm{CD}$ cases and 3 more in the non-CD group. These numbers, the limited numbers of reports and the lack of prevalence 
studies in the healthy population did not justify any conclusions of breed predisposition of $P$. carinii so far.

It has long been known that trimethoprim-sulfamethoxazole is effective against $P$. carinii (Hughes et al. 1975), and with the AIDS panepidemic many new drugs for PCP are in development. Even though most of the reported PCP cases in dogs have had a fatal outcome, responses to therapy have also been reported ( $\mathrm{Lo}$ betti et al. 1994). Therefore the clinician should also consider the possibility of PCP when treating secondary respiratory infections in $\mathrm{CD}$ dogs. Diffuse interstitial and bronchial densities in thoracic radiographs may indicate PCP and thus require more specific diagnostic procedures, such as broncho-alveolar lavatation with specific staining (Sukura et al. 1996), which has been shown to be a very good diagnostic method.

\section{A. Sukura ${ }^{l}, J$. Laakkonen ${ }^{l}$, and E. Rudbäck ${ }^{2}$. \\ ${ }^{1}$ Section of Anatomy, Faculty of Veterinary Medi- cine, University of Helsinki, and ${ }^{2}$ Pathology Unit, National Veterinary and Food Research Institute, Helsinki, Finland.}

\section{References}

Ainsworth DM, Weldon AD, Beck KA, Rowland PH: Recognition of Pneumocystis carinii in foals with respiratory distress. Equine vet. J. 1993, 25, 103108.

Bartlett MS, Smith JW: Pneumocystis carinii, an opportunist in immunocompromised patients. Clin. Microbiol. Rev. 1991, 4, 137-149.

Baskerville A, Dowsett AB, Cook RW, et al: Pneumocystis carinii pneumonia in simian immunodeficiency virus infection: immunohistological and scanning and transmission electron microscopical studies. J. Pathol. 1991, 164, 175-184.

Bille-Hansen V, Jorsal SE, Henriksen SA, Settnes OP: Pneumocystis carinii pneumonia in Danish piglets. Vet. Rec. 1990, 407-408.

Botha WS, Van Rensburg IBJ: Pneumocystosis: a chronic respiratory distress syndrome in the dog. J. S. Afr. vet. Assoc. 1979, 50, 173-179.

Farrow BRH, Watson ADJ, Hartley WJ, Huxtable
CRR: Pneumocystis pneumonia in the dog. J. Comp. Pathol. 1972, 82, 447-453.

Furuta T, Nogami S, Kojima $S$, et al: Spontaneus Pneumocystis carinii infection in the dog with naturally acquired generalised demodicosis. Vet. Rec. 1994, 134, 423-424.

Gardner MB, Luciw PA: Animal models of AIDS. The FASEB Journal 1990, 3, 2593-2606.

Grocott RG: A stain for fungi in tissue sections and smears. Am. J. clin. Pathol. 1955, 25, 975-979.

Hagler DN, Kim CK, Walzer PD: Feline leukemia virus and Pneumocystis carinii infection. J. Parasitol. 1987, 73, 1284-1286.

Hughes WT, Feldman S, Sanyal SK: Treatment of Pneumocystis carinii pneumonitis with trimethoprim-sulfamethoxazole. Can. med. Assoc. J. 1975, 112, 47-50.

Iwatsuki K, Okita M, Ochikubo F, Gemma T, Shin Y-S, Miyashita N, Mikami T, Kai C: Immunohistocehmical analysis of the lymphoid organs of dogs naturally infected with canine distemper virus. J. comp. Path. 1995, 113, 185-190.

Krakowa S, Higgins RJ, Koestner A: Canine distemper virus: review of structural and functional modulations in lymphoid tissues. Am J. vet. Res. 1980, 41, 284-292.

Laakkonen J, Soveri T: Characterization of Pneumocystis carinii infection in Sorex araneus from southern Finland. J. Wildl. Dis. 1995, 31, 228232.

Lobetti R, Leisewitz A, Spencer J: A preliminary investigation into the immunological basis of Pneumocystis carinii pneumonia in 4 dogs. WSAVA XIX World Congress Durban 1994, 77.

Mayhew IG, Greiner EC: Protozoal diseases. Vet. Clin. North. Am. Equine Pract. 1986, 2, 439-459.

McConnell EE, Basson PA, Pienaar JG: Pneumocystosis in a domestic goat. Onderstepoort J. Vet. Res. 1971, 38, 117-126.

Pedersen NC: The feline immunodeficiency virus. In: Levy JA (ed): The Retroviridae. v. 2. Plenum Press, New York, 1993.

Perryman LE, McGuire TC, Crawford TB: Maintenance of foals with combined immunodeficiency: causes and control of secondary infections. Am. J. vet. Res. 1978, 39, 1043-1047.

Phair J. Munoz A, Detels R, et al.: The risk of Pneumocystis carinii pneumonia among men infected with human immunodeficiency virus type I. N. Engl. J. Med. 1990, 322, 161-165.

Settnes OP, Hasselager E: Occurrence of Pneumocystis carinii Delanoe \& Delanoe, 1912 in dogs 
and cats in Denmark. Nord. Vet. Med. 1984, 36, 179-181.

Shimizu A, Kimura F, Kimura S: Occurrence of Pneumocystis carinii in animals in Japan. Jpn. J. vet. Sci. 1985, 47, 309-311.

Sukura A, Saari S, Järvinen A: Pneumocystis carinii pneumonia in dogs - a diagnostic challenge. $\mathbf{J}$. vet. diagn. Invest. 1996, 8, 124-130.

Vogel P, Miller CJ, Lowenstine LL, Lackner AA: Evidence of horizontal transmission of Pneumocys- tis carinii pneumonia in simian immunodeficiency virus-infected Rhesus Macaques. J. Infect. Dis. 1993, 168, 836-843.

Walzer PD: Pneumocystis carinii: recent advances in basic biology and their clinical application. Aids 1993, 7, 1293-1305.

Yoshida Y, Ikai T: Pneumocystis carinii pneumonia: epidemiology in Japan, and cyst concentration method. Zbl. Bakt. Hyg. I Abt. Orig. 1979, 244 , 405-410.

(Received November 11, 1996; accepted March 24, 1997).

Reprints may be obtained from: J. Laakkonen, Section of Anatomy, Faculty of Veterinary Medicine, P.O. Box 57, FIN-00014 University of Helsinki, Helsinki, Finland. E-mail: juha.laakkonen@helsinki.fi, tel: +358-9-708 49 796, fax: +358-9-708 49799 . 


